\title{
Experimental Testing and Modelling of a Variable Capacity Air-to-air Heat Pump in Cooling Mode
}

\author{
Gregor Strugala ${ }^{1}$, Michaël Kummert ${ }^{1}$, Martin Kegel ${ }^{2}$ \\ ${ }^{1}$ Polytechnique Montreal, Montreal, Canada \\ ${ }^{2}$ CanmetENERGY-Varennes, Varennes, Canada
}

\begin{abstract}
Due to their energy efficiency, air source heat pumps are an increasingly popular solution for space heating, despite their limitations in cold weather. Variable capacity heat pumps (VCHP) make use of variable speed inverter-driven compressors to improve comfort and to provide a broader operating temperature range encouraging larger evaporators/condensers to extract more energy from the air, without the downfall of being oversized at warmer conditions. This benefit has seen a growing interest for air source heat pump systems (ASHPs) in North America due to the wide operating conditions. While the variable capacity air-to-air (air-source) heat pumps (VCASHPs) are gaining popularity, there is still a lack of accurate performance curves and detailed models in building simulation tools in order to accurately assess their annual energy saving and utility cost reduction benefit. To address this gap, Natural Resources Canada, CanmetENERGY has been conducting both heating and cooling experimental tests of both ducted and ductless VCASHP systems to gain a better understanding of their performance and limitations as well as support the development of detailed performance models for building simulation tools. This paper presents the extension of a VCASHP TRNSYS heating mode component model, with the experimental testing of a ductless VCASHP in cooling mode under different operating conditions and steady state operation.
\end{abstract}

\section{Introduction}

Heat pumps are expected to play a significant role in improving the energy efficiency and the carbon footprint of the building sector in the context of decarbonized grids. Space heating and cooling represent a large fraction of the building energy consumption in most industrialized countries. For example, Natural Resources Canada (2018) estimates the share of space heating and cooling at $70 \%$ of the residential sector energy use.

In Canada, heat pumps can play a significant role in reducing the predominant space heating energy end use, in many instances consuming up to four times less energy than a conventional system utilizing the renewable energy resource. Air source and ground source heat pumps are the common systems each upgrading the available energy from the ambient air or ground, respectively. Naturally, air source heat pumps are a lower first cost option in comparison to ground source heat pumps; however their Coefficient of Performance (COP) and heating capacity decrease with lowering ambient temperature, so that auxiliary heating is often required. Furthermore, at warmer ambient conditions, the air source heat pumps deliver too much energy. Without thermal storage, constantspeed heat pumps must therefore cycle on and off to meet the load, leading to decreased performance and thermal comfort. This on/off cycling regime also precludes the selection of heat pumps able to meet the full load at low temperatures. To overcome this issue, variable speed compressors are now being used in many systems, offering the benefit of cycling to very low speeds to better meet occupant comfort and improved seasonal efficiencies.

As of now, VCHPs are difficult to model, for there is a lack of dynamic models able to predict accurately VCHPs behaviour and performance in a userfriendly way. Manufacturer data can be used, but they are not always easy to integrate into simulations, and are found to be incomplete and imprecise, which make them unreliable (US DOE, 2011). To facilitate the design of heating systems using VCHPS, they need to be adequately modelled, both in heating and cooling modes-better performance data is thus also necessary. In order to fulfil these objectives, a test bench was set up at Natural Resources Canada, CanmetENERGY in Varennes, to measure the performances of a variable capacity air-to-air (air source) heat pump (VCASHP) in different regimes of operation, and obtain more reliable results than those provided by the manufacturer. St-Onge et al. (2018) describe a VCASHP model that was developed for heating operation, and is able to predict performance degradation caused by defrost cycles accurately. The model presented in that paper is, however, limited to heating only and humidity is not taken into account in the performance maps. This paper focuses on ex- 
panding that model including a modelling strategy for cooling and taking humidity into account. The type of heat pumps that can be simulated by the model is thus restricted to air-to-air VCHPs, which usually require a lower investment cost than other HP types, such as ground-source HPs (Kegel et al., 2014). The concept can easily be expanded to other heat pump types in the future.

The article is divided into three sections. First, the test bench is described, along with the relevant variables measured, and how they impact the performances. Afterwards, results of the operation in cooling mode are presented, with the insights that they give on the control strategies. A preliminary performance map is compared to manufacturer data. Finally, the model is presented, and illustrated through some simulation results.

\section{Test bench}

Before introducing the test bench, namely what is measured and how, it is important to define what is to be measured, i.e. the quantities that must appear in the performance map, or that allow to obtain them through thermodynamic calculations.

The quantities provided by the performance map (the outputs, used by the model) are the total electric input power $\left(P_{\text {tot }}\right)$, the latent cooling capacity $\left(\dot{Q}_{\mathrm{cl}}\right)$ and the sensible cooling capacity $\left(\dot{Q}_{\mathrm{cs}}\right)$. The latter is necessary to compute the supply air temperature. The total cooling capacity $\left(\dot{Q}_{\mathrm{c}}\right)$ is not included, as it is simply obtained by $\dot{Q}_{\mathrm{cl}}+\dot{Q}_{\mathrm{cs}}$.

On the other hand, there are five quantities required by the performance map (which are included in the model inputs). First, the indoor and outdoor temperatures affect the heat transfer as they correspond to the heat source and sink, respectively. The outdoor temperature is noted $T_{\mathrm{o}}$, while the indoor temperature is noted $T_{r}$ as it is also the temperature of the return air entering the indoor unit. Another quantity that affects the heat transfer is the airflow rate; only the indoor air flow rate $\left(\dot{m}_{\mathrm{a}}\right)$ is considered because the outdoor air flow rate is not varying. Moreover, the power provided by the compressor depends on the frequency $(f)$ that the inverter is feeding to the compressor's motor. The frequency thus obviously has an impact on the capacity and the COP. Finally, the relative humidity of the return indoor air $\left(\phi_{r}\right)$ must be specified to predict the fraction of sensible and latent heat.

The aim of the test bench is to impose artificial environmental conditions to a VCASHP and measure the corresponding capacities and input power. The environmental test chamber is composed of two sheds in which the temperature - or the load - is controlled by a fan bringing air from outside, and/or an auxiliary heater. The fresh air and auxiliary heaters are controlled to meet desired test conditions (specific out-

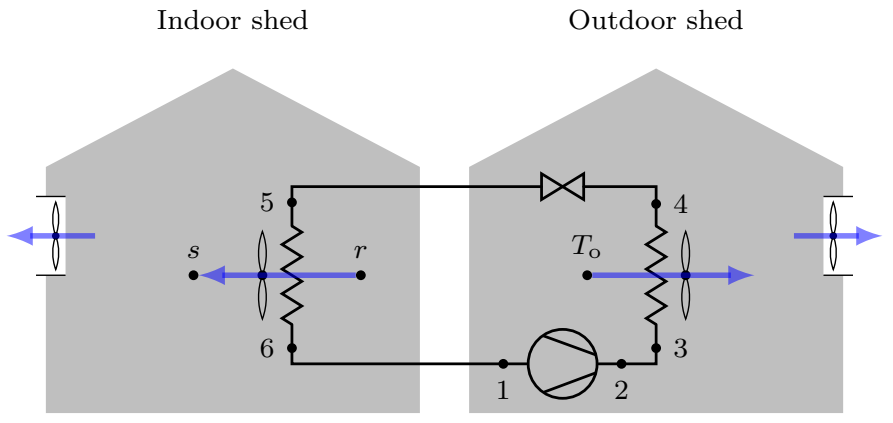

Figure 1: Representation of the sheds with the temperature sensors on the refrigerant lines.

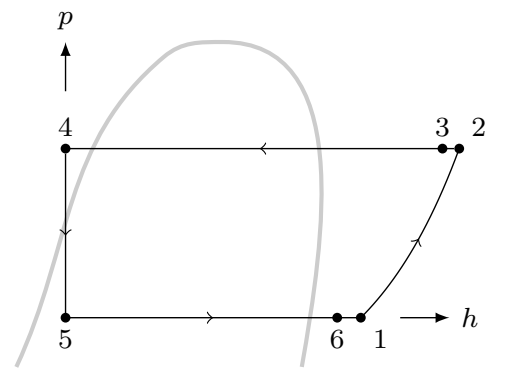

Figure 2: $p$-h diagram of the cycle.

door air temperature and relative humidity) counterbalancing the heat/cooling input from the heat pump. The outdoor shed contains the outdoor unit of the heat pump, and the imposed temperature is $T_{\mathrm{o}}$. In the indoor shed (where the indoor unit is located), the fan is used to impose a load (which should be equal to $\dot{Q}_{\mathrm{c}}$ in steady-state) that the heat pump must compensate to maintain the temperature setpoint $T_{r}$. The humidity of the return air is also measured, as well as the temperature and humidity of the supply air which are necessary to compute the capacitiessee details in the next paragraph. The refrigerant temperature is measured at several points along the HP cycle, as indicated in Figure 1. Each state number on the refrigerant lines corresponds to a temperature measurement. Additionally, the pressure of the refrigerant is measured at the inlet and outlet of the compressor (i.e. points 1 and 2), and its mass flow rate is measured using a coriolis flow meter. By neglecting pressure drops, the $p$ - $h$ diagram of the cycle will have a shape like the one depicted in Figure 2, where there are only two pressure levels, hence no need for additional pressure sensors. Further details of the test bench description can be found in St-Onge et al. (2018).

Among the variables introduced, $\dot{Q}_{\mathrm{cl}}$ and $\dot{Q}_{\mathrm{cs}}$ are not directly measured. They can be determined from $\dot{m}_{\mathrm{a}}$ and the temperature and humidity of the return $(r)$ and supply $(s)$ air. With the conditions $\left(T_{r}, \phi_{r}\right)$ and $\left(T_{s}, \phi_{s}\right)$, the enthalpies $h_{r}$ and $h_{s}$ are determined using humid air properties (we assume a constant pres- 
sure, $p_{r}=p_{s}=1 \mathrm{~atm}$ ), then the capacities are given by

$$
\begin{aligned}
& \dot{Q}_{\mathrm{cl}}=\dot{m}_{\mathrm{a}}\left(h_{r}-h_{x}\right) \\
& \dot{Q}_{\mathrm{cs}}=\dot{m}_{\mathrm{a}}\left(h_{x}-h_{s}\right)
\end{aligned}
$$

where $x$ is the state where $T_{x}=T_{r}$ and $\omega_{x}=\omega_{s}$. ( $\omega$ designates the absolute humidity.) However, measurements of the air flow rate are found to be less accurate than those of the refrigerant flow rate, and the composition of the refrigerant does not vary, which means that the capacity can be calculated from temperatures and flow rate only, and is thus less prone to errors. For these reasons, $\dot{Q}_{\mathrm{c}}$ is calculated using the refrigerant properties, and $\dot{Q}_{\mathrm{cs}}$ using

$$
\dot{Q}_{\mathrm{cs}}=\dot{Q}_{\mathrm{c}} \frac{h_{x}-h_{s}}{h_{r}-h_{s}}
$$

The air-side cooling capacity $\dot{Q}_{\mathrm{c}}$ corresponds to the heat extracted by the evaporator and the condensing water (if any), minus the power of the indoor unit fan, which heats up slightly the cooled air:

$$
\dot{Q}_{\mathrm{c}}=\dot{Q}_{\mathrm{ev}}+\dot{m}_{\mathrm{w}} h_{\mathrm{w}}-P_{\mathrm{fan}, \mathrm{i}}
$$

Since the condensate flow rate $\dot{m}_{\mathrm{w}}$ is very small, its influence is neglected. The fan power is directly measured, while $\dot{Q}_{\mathrm{ev}}$ needs to be calculated using

$$
\dot{Q}_{\mathrm{ev}}=\dot{m}_{\mathrm{r}}\left(h_{6}-h_{5}\right)
$$

where $\dot{m}_{\mathrm{r}}$ is the refrigerant mass flow rate. The refrigerant in state 6 is a superheated gas, which means that $h_{6}$ can be deduced from $p_{6}$ and $T_{6}$ only, however in state 5 it is a liquid-gas mixture, for which the quality is unknown. Without a way to compute adequately $h_{5}$, an isenthalpic expansion is assumed between states 4 and 5, as depicted in Figure 2. An easier way to obtain $\dot{Q}_{\mathrm{ev}}$ is then

$$
\dot{Q}_{\mathrm{ev}}=\dot{m}_{\mathrm{r}}\left(h_{6}-h_{4}\right)
$$

where $h_{4}$ can be determined from $p_{4}=p_{2}$ and $T_{4}$ only, as the refrigerant in state 4 is subcooled.

Moreover, we can also compute the heat rejection at the condenser as well as the power transmitted by the compressor to the refrigerant.

$$
\begin{aligned}
& \dot{Q}_{\text {cond }}=\dot{m}_{\mathrm{r}}\left(h_{3}-h_{4}\right) \\
& P_{\text {comp }}=\dot{m}_{\mathrm{r}}\left(h_{2}-h_{1}\right)
\end{aligned}
$$

Now that every relevant quantity can be evaluated, it is important to determine what measurements are adequate to be integrated in the performance map. Some measurements should be excluded because they represent short-term transients that cannot be captured by the model. One way to do that is to compare $P_{\text {comp }}$ to the electrical power consumed by the compressor, $P_{\mathrm{el}}$. Indeed, both electrical and thermal losses cause a difference between the two, but this difference is found to be much more important during transient regimes (this is illustrated in the next section). The relative difference between $P_{\text {comp }}$ and $P_{\mathrm{el}}$ is given by

$$
\delta=\left|1-\frac{P_{\text {comp }}}{P_{\text {el }}}\right|
$$

When the compressor starts up, $\delta=100 \%$ and then decreases steadily to stabilize around $20 \%$. Accounting for small variations, measurements are thus considered valid if $\delta<30 \%$, that is, if

$$
\left|P_{\text {el }}-P_{\text {comp }}\right|<0.3 P_{\text {el }}
$$

\section{Results}

The tested heat pump has two modes for the fan operation: a "fixed-speed" mode where the user can choose between high, medium, low and quiet, and an "auto" mode where the controller chooses one of those four settings. It was found that the heat pump is unable to properly modulate the frequency while being in the "fixed-speed" mode, as shown in Figure 3a. Instead, it operates with on-off cycles like a classic heat pump, except that the machine cycles between off and a fixed part-load capacity - which still reduces the cycling frequency compared to a classic heat pump.

Figure $3 \mathrm{~b}$ shows the operation at full capacity. The relative difference between $P_{\mathrm{el}}$ and $P_{\text {comp }}, \delta$, is depicted on the bottom plot. It is not under $30 \%$ during the first half-hour, where there appears to be a transient process causing a big gap between $P_{\mathrm{el}}$ and $P_{\text {comp }}$. This difference could have several causes not considered by the model: the thermal mass of the compressor, the influence of the lubricant mixed with the refrigerant, and the fact that the refrigerant is not necessarily in the "right" state at start-up. When investigating the refrigerant properties, this relatively slow transient evolution is clearly seen in the pressure and the temperature of state 2 , at the compressor outlet. The slow evolution of $T_{2}$ is likely the result of thermal mass in the system and dynamic processes in the refrigerant. After a half-hour, both $p_{2}$ and $T_{2}$ flatten out, and a steady-state is reached. At that point, $P_{\mathrm{el}}$, and $P_{\text {comp }}$ are closer (with a difference of about $20 \%$ ).

With the "auto" mode however, measurements show some frequency modulation to match the loadthough still not as smooth as one would get with a PID controller. Rather, there seems to be discrete frequency levels at which the controller chooses to operate. Figure 4 clearly shows steps of about $7 \mathrm{~Hz}$, and the capacity varies accordingly, to try and match the load. It is however not very clear what the frequency levels are, as in the second half of the test, there are narrow peaks reaching the maximum frequency. The minimum frequency announced by the manufacturer is $10 \mathrm{~Hz}$, however the controller does not seem to be 


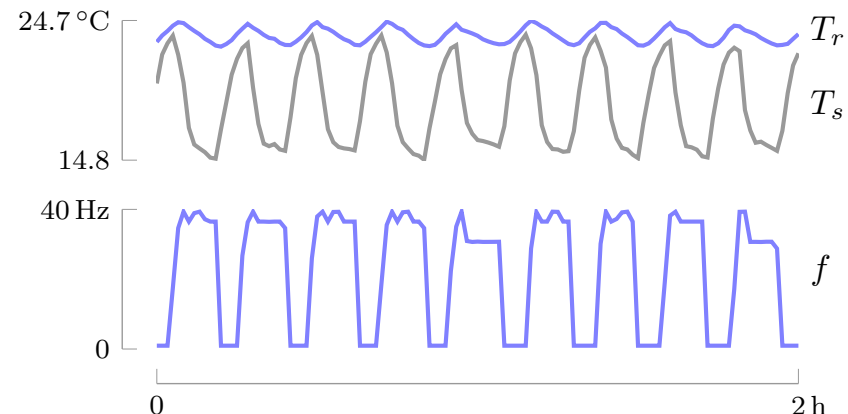

(a) Operation at $65 \%$ of the rated capacity.
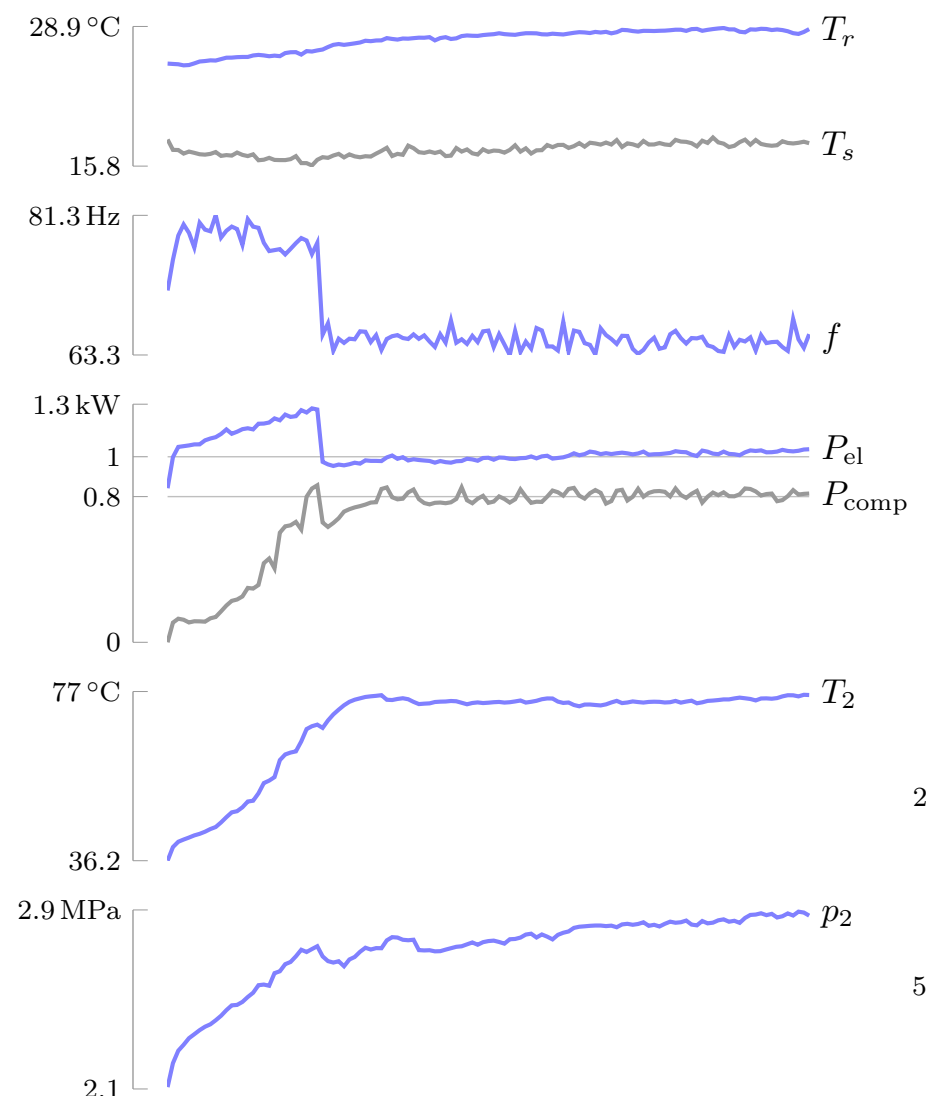

$5.2 \mathrm{~kW}$

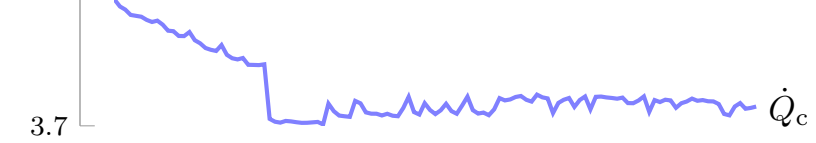

$100 \%$

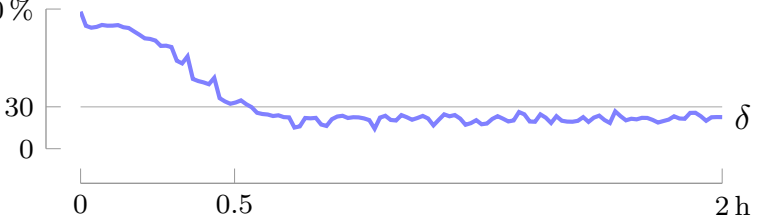

(b) The load exceeds the capacity. The frequency step at $0.5 \mathrm{~h}$ seems to be triggered when the quantities $p_{2}$ and $T_{2}$ reach their steady-state value.

Figure 3: Results with the "fixed-speed" mode over a two-hour period.
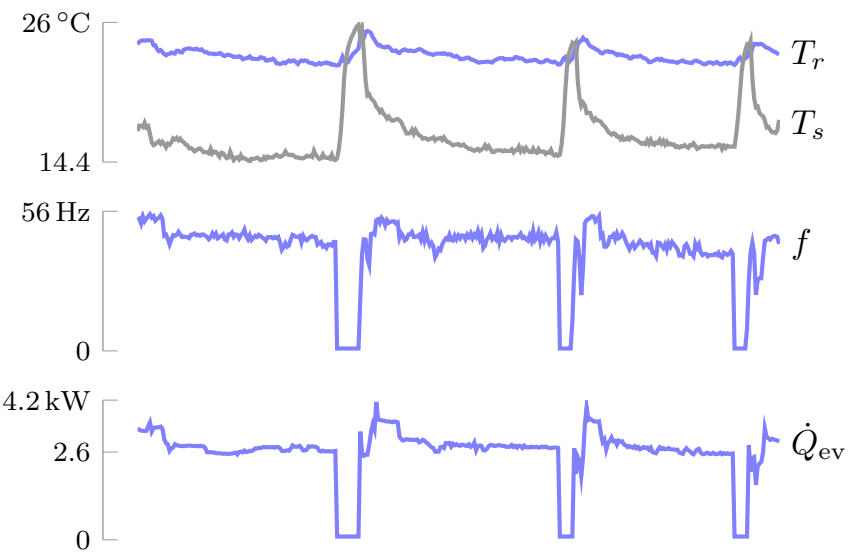

$0.8 \mathrm{~kW}$

Figure 4: Results using the "auto" mode at $85 \%$ of the rated capacity.

Rome, Italy, Sept. 2-4, 2019 
able to decrease the frequency lower than $40 \mathrm{~Hz}$ without turning off, as shown in Figure 5. A possible cause is that the compressor power is relatively low, and it may not be able to operate properly at such lower power levels.

Control strategies implemented by VCASHP manufacturers are proprietary, so modeling them requires reverse engineering using the observed behavior. The base control law is a variant of conventional PI or PID algorithms, but the controllers have additional rules that seem to aim at maximizing comfort (e.g. during start-up or ramp-up phases) and protecting the machine (by limiting temperatures, pressures, or current). In addition to the compressor speed, the controller also adjusts the fan speed (in "auto" mode) and performs other operations such as defrost cycles in heating mode. In order to isolate the control complexities from the thermal performance aspects, the controller model was separated from the performance model, allowing different control strategies to be compared for the same heat pump.

As explained in the previous section, the test bench depicted in Figure 1 allows to control the temperature easily, however it faces challenges when it comes to humidity control. When needed, the fan brings fresh air to keep the humidity constant, however the humidity ratio (HR) cannot be higher than that of the outside air, so there is an upper bound on HR values that can actually be reached. At the time of writing, experimental results were only obtained at low humidity, hence manufacturer data are used to complete the performance map for higher HR values. Another thing to bear in mind regarding the performance map is that the compressor's frequency cannot be explicitly controlled like other quantities such as the outdoor temperature, for it is imposed by the controller depending on those other quantities - for this reason, a color map is used for the frequency in Figure 6. While the other input variables of the performance map are independent - except for $\dot{m}_{\mathrm{a}}$ in "auto" mode - all combinations of the selected values for those variables do not need to be tested, since some of them correspond to impossible or very unlikely situations, such as a very low temperature combined with a very high humidity content.

It can be seen in Figures 6 and 7 that manufacturer data are provided for very cold temperatures in cooling mode, down to $-10{ }^{\circ} \mathrm{C}$. However, assessing performance at such low temperatures is not really relevant for air conditioning in the residential sector. In the temperature range where experiments were carried out, the experimental results and the manufacturer data show similar trends, even if not enough data are available at this point for a complete validation. The measured total input power is more spread out than the manufacturer data, however the air flow rate is not the same for every point, while it is kept

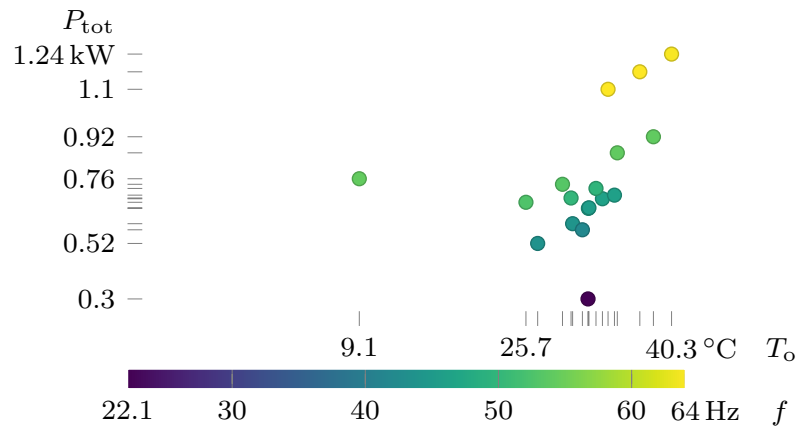

(a) Experimental data.

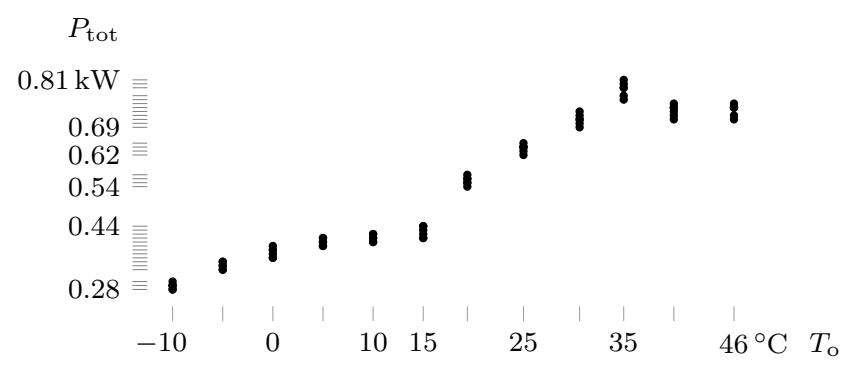

(b) Manufacturer data (Fujitsu, 2012).

Figure 6: Measured total input power vs outdoor air temperature.

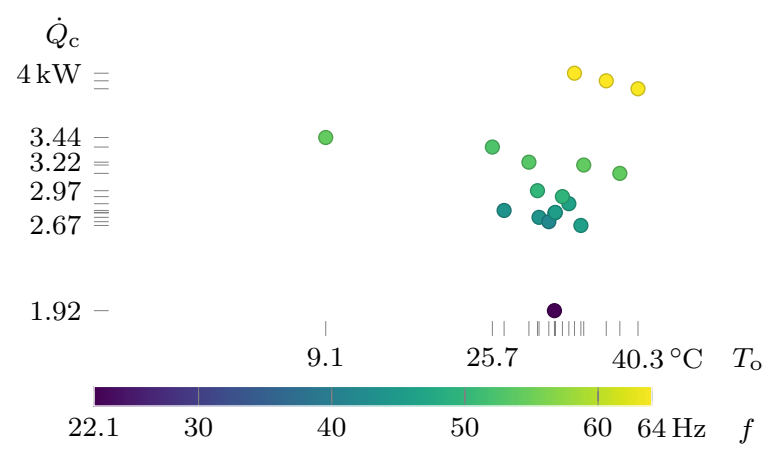

$\dot{Q}_{\mathrm{c}}$

(a) Experimental data.

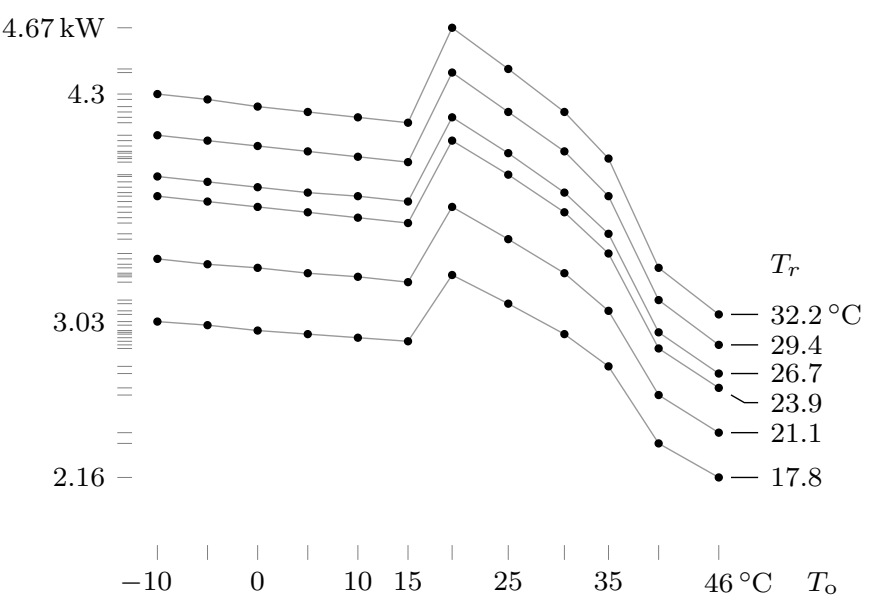

(b) Manufacturer data (Fujitsu, 2012).

Figure 7: Measured total capacity vs outdoor air temperature. 


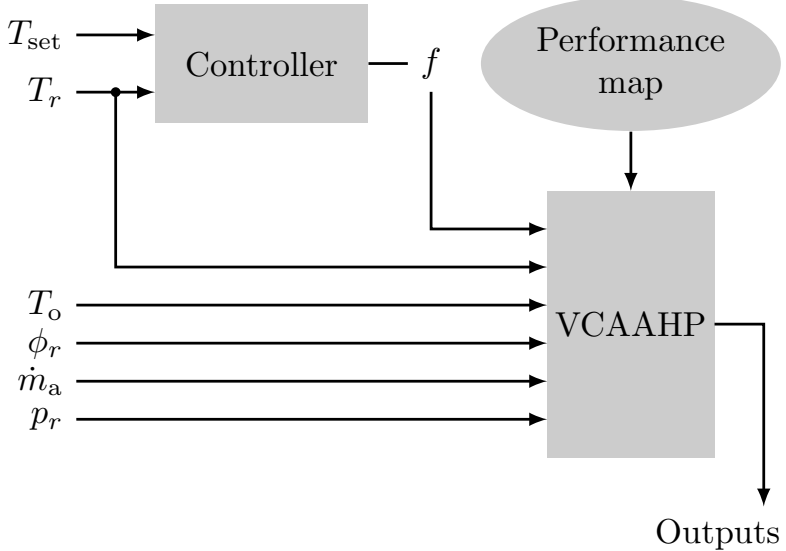

Figure 8: TRNSYS model overview. The controller and the VCAAHP itself are implemented as different components.

to $830 \mathrm{~m}^{3} \mathrm{~h}^{-1}$ (high mode) in Figures $6 \mathrm{~b}$ and $7 \mathrm{~b}$.

\section{Model}

The main objective of the model is, given a set of inlet conditions, to compute the different performance variables $\left(\dot{Q}_{\mathrm{c}}, \dot{Q}_{\mathrm{cs}}, \dot{Q}_{\mathrm{cl}}, P_{\text {tot }}, \mathrm{COP}\right)$ and to compute the outlet conditions $\left(T_{s}, \phi_{s}\right)$. The formers are determined thanks to the performance map, and they allow to compute the latters. Indeed, once they are known, the only unknowns left in equation (1) are $h_{s}$ and $h_{x}$. The supply humidity ratio $\omega_{s}=\omega_{x}$ can be determined from $h_{x}$ and $T_{x}=T_{r}$. By neglecting the (very small) pressure drop caused by the fan, $T_{s}$ and $\phi_{s}$ are obtained from $h_{s}, \omega_{s}$ and $p_{s}=p_{r}$. Note that if $\dot{Q}_{\mathrm{cl}}=0 \mathrm{~W}$ (i.e. $\dot{Q}_{\mathrm{cs}}=\dot{Q}_{\mathrm{c}}$ ), unnecessary calculations can be avoided by simply setting $\omega_{s}=\omega_{r}$. From $\omega_{s}$, the condensate mass flow rate is obtained using $\dot{m}_{\mathrm{w}}=\dot{m}_{\mathrm{a}}\left(\omega_{r}-\omega_{s}\right)$. Finally, the COP is directly given by

$$
\mathrm{COP}=\frac{\dot{Q}_{\mathrm{c}}}{P_{\mathrm{tot}}}
$$

As mentioned above, since control strategies may vary significantly from one manufacturer to another, that part is not embedded in the model itself. Rather, a separate TRNSYS component computes the frequency and feeds it to the heat pump model (see Figure 8), so that the user can easily switch to another one (possibly custom) if needed. Some users may also need to use $\omega_{r}$ instead of $\phi_{r}$ to quantify the humidity, so a parameter allows to choose which input should be used.

Trying to supplement experimental data with manufacturer data represents a challenge, as published data in the different manuals and specification documents is very often incomplete. The data in Figures $6 \mathrm{~b}$ and $7 \mathrm{~b}$ are for constant compressor frequency and air flow rate, and the indoor dry-bulb and wetbulb temperatures are varied together (so that only

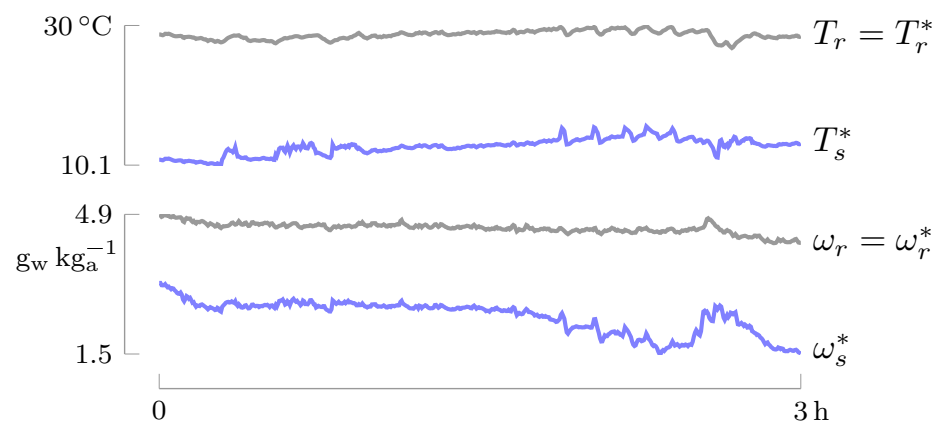

(a) Simulated temperature and humidity.

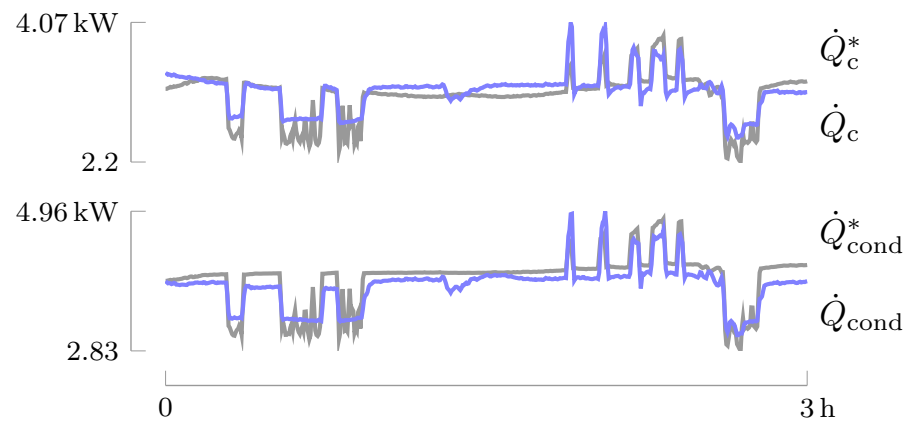

(b) Heat absorbed and rejected.

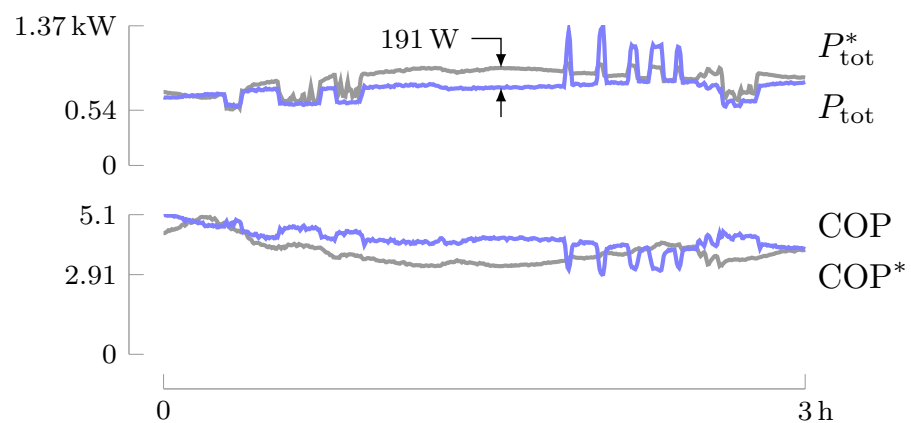

(c) Input power and $C O P$.

Figure 9: Comparison between measurements and simulation. Simulated quantities are designated by a superscript ${ }^{*}$ and plotted in gray. 
one humidity level is provided for a given temperature level). In order to test the model without waiting for a complete experimental performance map, additional performance data for the same machine were obtained from a published third-party test report (US DOE, 2011). Further correction for different humidity levels and variable frequency were obtained from other manufacturer data for comparable machines. The resulting composite performance map does not aim to represent the tested machine, but is simply used to illustrate how the model would operate with a complete performance map.

Figure 9 shows simulation results obtained using the measurements of the test displayed in Figure 4 as inputs. The simulated total cooling capacity matches quite well the measurements, while the total input power shows a more important gap going up to $14 \%$ of the peak value. This difference has repercussions on the COP, for which the difference between measurements and simulation is more significant when the measured and simulated power differ as well. The measured supply air temperature and humidity are not displayed in Figure 9, because the measurements during the reported tests were not usable (showing physically impossible conditions). This problem has not been addressed yet and needs further investigation.

\section{Conclusion}

VCASHP performance depends on several environmental and control variables, increasing the complexity of the performance data (often expressed as performance maps) that must be used to define a simulation model of the equipment.

This paper describes a test bench that was developed to obtain performance data for different VCASHPs in heating and cooling modes, over a large operating range. The paper presents partial cooling data which, when completed by additional tests, will be used to develop a complete performance map and TRNSYS VCASHP component model. These results show that the controller mode has a large impact on the operating regimes of the compressor, completely preventing frequency modulation in some cases. A TRNSYS model has been developed for the heat pump and for the controller. The TRNSYS model is exercised with a performance map based on partial experimental data supplemented with manufacturer data. Further work will aim at completing the performance map and demonstrating the use of the complete model.

\section{References}

Fujitsu General Limited (2012). Service Instruction. Model: ASU12RLS3.

Kegel, M., J. Tamasauskas, R. Sunye, and D. Giguère (2014). Heat Pumps in the Canadian Residential sector. In IEA HPP Conference.
Natural Resources Canada (Ottawa, 2018). Secondary Energy Use and GHG Emissions by EndUse in 2015-2016 for the Residential Sector. Accessed January 18, 2019.

St-Onge, G., M. Kummert, and M. Kegel (2018). Variable capacity mini-split air source heat pump model for TRNSYS. In eSim 2018, the 10th conference of IBPSA-Canada, Montreal, QC, CAN, May 9-10.

US Department of Energy (Oakridge, TN, USA, 2011). Laboratory Test Report for Fujitsu 12RLS and Mitsubishi FE12NA Mini-Split Heat Pumps. 